
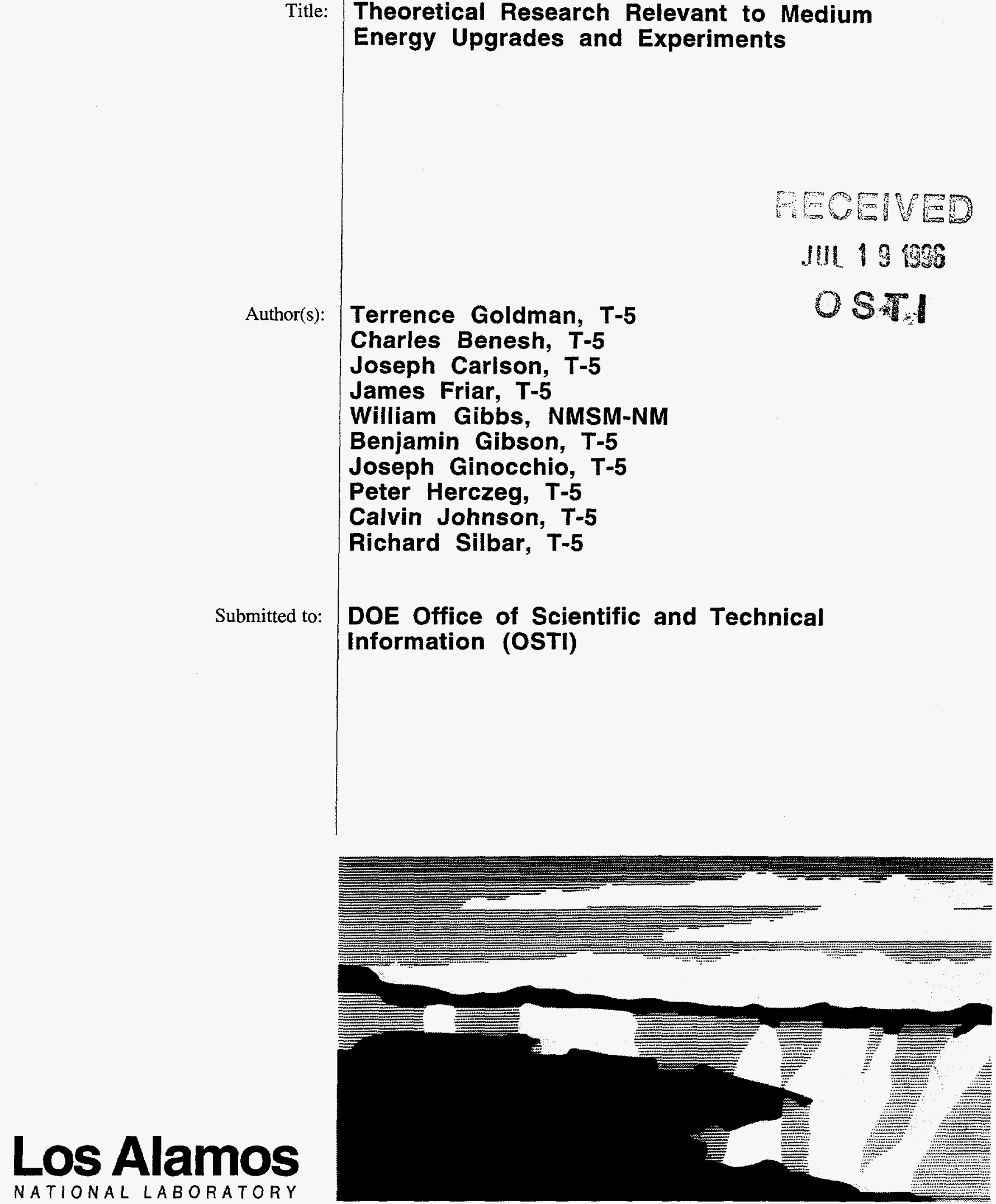

Los Alamos National Laboratory, an affirmative action/equal opportunity employer, is operated by the University of California for the U.S. Department of Energy under contract W-7405-ENG-36. By acceptance of this article, the publisher recognizes that the U.S. Government retains a nonexclusive, royaltyfree license to publish or reproduce the published form of this contribution, or to allow others to do so, for U.S. Government purposes. The Los Alamos National Laboratory requests that the publisher identify this article as work performed under the auspices of the U.S. Department of Energy. 


\section{DISCLAIMER}

Portions of this document may be illegible in electronic image products. Images are produced from the best available original document. 


\title{
Theoretical Research Relevant to Medium Energy Upgrades and Experiments
}

\author{
Terrence Goldman*, Charles Benesh, Joseph Carlson, James Friar, \\ William Gibbs, Benjamin Gibson, Joseph Ginocchio, Peter Herczeg, \\ Calvin Johnson, and Richard Silbar
}

\begin{abstract}
This is the final report of a three-year, Laboratory-Directed Research and Development (LDRD) project at the Los Alamos National Laboratory (LANL). This work provides theoretical research results for sources of pions, neutrons, neutrinos and heavy ions. We have undertaken specific calculations in neutrino-nucleus scattering and to analyze constraints on exotic decays relevant to the study of neutrino oscillations. We have also performed calculations regarding neutrino cosmology and astrophysics relevant to the experimental study of neutrino masses. We analyzed the constraints of data on T-violation in neutron decay and the nucleon-nucleon (NN) interaction which will be important for the understanding of experiments with cold and ultra-cold neutrons. We completed several specific calculations which were essential to predict (pion, kaon) hypernuclear production and pion-induced reactions studying baryonic resonances. We also calculated the nuclear spectroscopy of nuclei far from stability that can be studied experimentally.
\end{abstract}

\section{Background and Research Objectives}

Our work has provided calculations and prediction of key parameters of importance to experimenters who are evaluating various potential facility upgrades for sources of pions, neutrons, neutrinos and heavy ions. We have carried through specific calculations in neutrino-nucleon scattering and analyzed constraints on exotic decays relevant to the large scintillating neutrino detector (LSND) and the pulsed lepton source (PLS) upgrade of the Los Alamos Meson Physics Facility (LAMPF). We also performed calculations regarding available fluxes and spectral shapes which have implications for various Los Alamos *Principal investigator, e-mail: tgoldman@lanl.gov 
neutrino experiments relevant to neutrino masses. For proposed and upcoming Los Alamos experiments with neutrons at Grenoble, LANSCE/WNR, and an ultra-cold neutron source at LANL, we analyzed the constraints of data on T-violation in neutron decay and the nucleon-nucleon (NN) interaction.

\section{Importance to LANL's Science and Technology Base and National R\&D Needs}

Our work is in the area of medium energy theory. The distinction between nuclear and particle physics is blurred in this area. High precision, lower-energy experiments have become essential for clarifying the underlying fundamentals of particle physics and establishing limits on alternative explanations. At the same time, high energy experiments and theory have come to play an important role in nuclear physics. Our work is at the forefront of this interface between the two fields and has special emphasis on problems relevant to possible future upgrades at LAMPF and other medium energy facilities, such as the Brookhaven National Laboratory (BNL) Relativistic Heavy Ion Collider (RHIC), the Colliding Electron Beam Accelerator Facility (CEBAF), the Indiana University Cyclotron Facility (IUCF) and the Tri-Universities Meson Facility (TRIUMF/KAON) which are of interest to LANL researchers.

a. Inclusive Scattering and Euclidean Response. We have continued and extended our studies of inclusive scattering on light nuclei, using Path-Integral techniques to obtain the Euclidean (imaginary-time) response. The calculations are relevant to a large number of experiments, both at electron scattering machines (Massechusetts Institute of Technology Bates Laboratory and CEBAF) and in hadronic probes (LAMPF). Only through studying a variety of processes can we obtain a coherent picture of the dynamics probed in inclusive scattering.

b. Electromagnetic Dissociation in Heavy-Ion Collisions. The availability of relativistic heavy-ion beams has opened a new avenue for the study of electromagnetic excitations of nuclei. Experiments range from single and double nucleon-removal reactions, to the study of Shalom nuclei using radioactive beams, to the possibility of multi-photon excitations of collective nuclear states. Aside from their intrinsic interest, electromagnetic excitation processes in peripheral collisions are important for determining the beam lifetime at RHIC. This facility 
will be one of the mainstays of experimental nuclear physics in the U.S. in the next century, and of interest to LANL experimentalists.

c. Strangeness in Nuclei. Hypernuclear research, which is relevant to experiments at BNL involving Los Alamos staff, is motivated by the fact that the physics of hypernuclei clearly differs from the physics of conventional nuclei. In particular, the interactions of hyperons in matter differ significantly from the interactions of nucleons. Few-baryon systems provide filters through which one can look at particular aspects of the hyperon-nucleon force. They yield constraints on models of the interaction and are particularly sensitive to $\Lambda \mathrm{N}-\Sigma \mathrm{N}$ coupling and its induced spin dependence. Furthermore, extrapolating our experience to the strangeness -2 sector leads us to predict strong suppression of $\Lambda \Lambda-\Xi N$ coupling in all but the lightest of the $\Lambda \Lambda$ hypernuclei.

\section{d. Nuclear Structure with Pion Double Charge Exchange.} Having finished testing generalized seniority using LAMPF double-charge exchange experiments on the nickel isotopes and having predicted the generalized seniority cross section for the tin isotopes, we are calculating double charge exchange for other isotopes in the tin region. In particular we are studying the pion double-charge-exchange cross section for the tellurium isotopes. These isotopes are some of the very few that can double beta decay. Since double charge exchange to the ground state of xenon with tellurium as the target has the same initial and final state as double beta decay of tellurium, this reaction can test the wave functions used to calculate double beta decay.

e. New Collective Proton Rich Nuclei. Most heavy collective nuclei have a large neutron excess and hence collective motion can ignore the isospin quantum number. However, the production of heavy nuclei with the number of neutrons and protons roughly equal, as would be the case at the new radioactive beam facility at Oak Ridge or at the IsoSpin Laboratory (ISL) that has been under consideration for possible construction at LANL, or any other radioactive beam facility, necessitates that isospin be included in any description of collective motion.

\section{f. Neutron Proton Symmetry in Collective Motion. Nuclear} collective motion in nuclei is usually assumed to be symmetric in the neutron and proton degrees of freedom. The interacting boson model has a quantum number called F-spin which measures to what extent collective motion is symmetric. We previously determined the extent of this symmetry for ${ }^{165}$ Ho using LAMPF single- 
charge-exchange experiments on an oriented Ho target and have suggested other measurements; for example, the magnetic moment of the first and second excited $2^{+}$states.

\section{g. P-Conserving T-Violation in Flavor Conserving Quark-}

Quark Interactions. We have continued the investigation of parity-conserving time-reversal violation (PCTV) in flavor-conserving quark-quark interactions. The study of such interactions is of relevance to experiments searching for PCTV in hadronic nuclear processes (e.g. in nuclear gamma decay or in neutron transmission).

h. Further Effects of Leptoquarks in Beta Decay. We have investigated the effects of leptoquark exchange on time-reversal even beta decay observables, and analyzed the constraints on the corresponding interactions. In addition to $\mathrm{V}, \mathrm{A}$ interactions, spin-one and spin-zero leptoquark exchange generates scalar (S) type beta decay interactions, and spin-zero leptoquark exchange also generates tensor $(T)$ type beta decay interactions.

\section{Scientific Approach and Results}

a. The T-Odd D-Coefficient in Beta Decay. New sensitive experiments are in preparation at the National Institute for Standards and Technology (NIST) (with Los Alamos participation) and at Princeton to search for the T-odd correlation $\langle J\rangle$. $p_{e} x p_{V} / J E_{e} E_{V}$ (where $\underline{J}$ is the nuclear spin) in beta decay. We have investigated the coefficient $D$ of this correlation in next to leading order in the new interactions. This extends our previous work on the $D$ coefficient. The important term is the contribution from interactions involving the right-handed electron neutrino $v_{e}{ }^{(R)}$. This contribution arises from the interference of the amplitudes generated by four-fermion couplings containing the right-handed and the left-handed quark currents, respectively. We have investigated this contribution in extensions of the standard model where the $D$ coefficient can arise at the tree level. We have found that while this term can be neglected in both leftright symmetric models and in models with exotic fermions, in models where the new $\mathrm{V}, \mathrm{A}$ interactions arise from the exchange of leptoquarks the contribution from $v_{e}{ }^{(R)}$ can be as large as the one from the left-handed neutrino.

b. Constraints on Leptoquarks from the $\pi \rightarrow$ eve $/ \pi \rightarrow$ $\mu v_{\mu}$ Ratio. Recently we have investigated the constraints on pseudoscalar-type 
$\underline{d} u->e v_{e}$ interactions from the $\pi \rightarrow e v_{e} / \pi \rightarrow \mu v_{\mu}$ branching ratio $R \pi$. The ratio $R_{\pi}$. is especially sensitive to such couplings, since their contribution to the $\pi$ $\rightarrow e v_{e}$ amplitude has a large enhancement factor. Limits on pseudoscalar-type $\underline{d} u$ $\rightarrow e v_{e}$ interactions have been derived in previous works under the assumption that their contribution is small relative to the contribution from the Standard Model. We pointed out that for some important cases this assumption is not justified, and gave an exact treatment of the constraints from $R_{\pi}$. This yielded upper limits on pseudoscalar couplings that are larger than what follows from the previous treatments by a factor of $\sim 400$.

We have now analyzed the implications of the above results for the masses and couplings of leptoquarks. Leptoquarks are bosons that couple to quark-lepton pairs. They are present in many extensions of the Standard Model. Currently there is special interest in leptoquarks that couple to the first fermion family since sensitive direct searches became possible at the electron-positron collider HERA at DESY. In this connection the leptoquarks of interest are those that are not severely constrained by indirect bounds on the masses and couplings. Thus leptoquarks that couple to both left-handed and right-handed quarks (nonchiral leptoquarks) have been excluded from consideration since the exchange of such leptoquarks generates pseudoscalar-type couplings contributing to $\pi->e v_{e}$, for which the stringent constraints from $R_{\pi}$. were believed to apply. We have pointed out that with the exact treatment of the constraints from $R \pi$. the bounds on the masses of some of the nonchiral leptoquarks are not considerably stronger than the bounds on the masses of chiral leptoquarks even for equal strength for the left-handed and the right-handed couplings.

\section{c. Parity Conserving Time Reversal Violation in Flavor-}

Conserving Quark-Quark Interactions. We have continued work on Pconserving $\mathrm{T}$-violating (PCTV) flavor-conserving ( $\Delta f=0) q-q$ interactions.

We have found a more transparent and more compact proof of our theorem (discovered earlier), which states that such interactions are absent in any renormalizable gauge theory to order $f^{2}$ and to all orders in the CP-invariant part of the QCD and the QED interactions. Here $f_{Y}$ denotes generically the coupling constants of the massive bosons to the fermions and the coupling constants among the massive bosons. We also have studied the general structure of the PCTV $(\Delta f$ =0) $q-q$ interactions (which can be induced in higher order) and found that, in addition to the PCTV interactions considered in the literature, two further 
interaction terms have to be included in a general treatment. These terms make it possible to induce PCTV in the $(\Delta f=0) q-q$ interactions in new ways.

d. Origin Of Confinement Related to Glueballs. We showed that the coupling of a composi interaction to the interior of hadrons. By using crossing symmetry, we were able to extend this to a description of the expected spatial structure of such glueballs and delineate expectations for their experimental observation.

e. Systematic Search for Dibaryons in SU(3) FLAvor Space. We completed our study of baryon-baryon scattering amplitudes in relativistic and non-relativistic models of the strong interaction based on quark substructure, using generalized fractional parentage expansion coefficients, including varying the allowed basis of states for the descriptions. Center-of-mass motion was corrected for, and $\mathrm{P}$-waves were studied. The results show that over a wide range of models of the dynamical mechanisms, all of which agree well with measured scattering amplitudes where available, the same few strange and non-strange dibaryons are predicted with very little variation in the expected mass value. Experimental interest has been piqued to the extent of designing specific experiments which will require calculation of angular production cross sections.

f. Possible Mechanism for Gamma Ray Bursts. We identified the Casimir effect in quantum field theory as a possible mechanism for gamma ray bursts originating from starquakes on neutron stars. The motion of dielectric relative to vacuum converts the gravitational energy into a uniquely flat spectrum up to a frequency characteristic of nuclear media. In addition to matching the astrophysical observations, we estimated a close match between the energy produced and that derived for the source from observations, thus supporting our hypothesis.

\section{g. Testing Generalized Seniority with Pion Double Charge \\ Exchange.}

Generalized seniority assumes that the ground state of a semi-magic nucleus is a condensate of correlated pairs of valence nucleons. It is a generalization of seniority, which is applicable to valence nucleons in a single-shell-model orbital, to heavy nuclei for which the valence nucleons are filling many orbitals. The double charge exchange (DCX) reaction is unique in that it takes place, in lowest order, on a pair of nucleons. The pion is the least complex probe with which DCX is possible. Hence pion DCX is a good reaction to determine whether generalized seniority is valid. 
We have derived the DCX cross section to the double isobaric analog state (DIAS) in the generalized seniority model as a function of the number of valence nucleons. We have shown that it depends on only two complex amplitudes (three real numbers, since the cross section is the absolute magnitude of the total amplitude). Hence, we have more than three targets and so can determine the amplitudes and also test whether generalized seniority is valid. We have used recent measurements at LAMPF of the $95 \mathrm{MeV}$ pion DCX cross sections on the nickel isotopes to confirm that generalized seniority is valid for these nuclei and to determine the two complex amplitudes.

We also derived the DCX cross section to the ground state of the neighboring zinc isotopes. We showed that, unlike the seniority model, this cross section also depends on two complex amplitudes, one of which has been determined by the transition to the DIAS. We compared the ground state cross sections measured at LAMPF and extracted this additional amplitude but showed that any conclusions about the validity of generalized seniority for the zinc isotopes is ambiguous because of the large experimental errors on the cross sections.

We have also calculated the DCX cross sections to the DIAS and the ground state for $5 \mathrm{MeV}$ pions where experience on the calcium isotopes has taught us that these cross sections are larger than at $9 \mathrm{MeV}$. We have done this for the tin isotopes as well as the nickel isotopes since there are many more tin isotopes available as targets and hence a more stringent test of generalized seniority would be possible.

We have calculated double charge exchange for other isotopes in the tin region, in particular, the tellerium isotopes. These isotopes are some of the very few that can double beta decay. Since double charge exchange to the ground state of xenon with tellerium as the target has the same initial and final state as double beta decay of tellerium, this reaction can test the wavefunctions used to calculate double beta decay. We hope these calculations will motivate future DCX experiments, either with pions or heavy ions.

\section{h. Neutron-Proton Symmetry and F-Spin Admixtures in}

Nuclei. A new quantum number in nuclear physics called F-spin measures the degree to which collective motion is symmetric under the interchange of neutrons and protons. This quantum number is different than isospin, which relates the ground state of one nucleus to an excited state in the neighboring isobar, the isobaric analog. In contrast F-spin relates the ground states of different isobars and 
has its maximum value when the collective motion is completely symmetric in the neutron and proton degrees of freedom. We have shown that the admixture of lower values of F-spin in the ground state can be determined by measuring the difference in the neutron and proton deformation in nuclei. Using data from a LAMPF pion single-charge-exchange experiment on aligned ${ }^{165} \mathrm{Ho}$, we determined that only 6-nucleus of lower F-spin values for this well-deformed nucleus are mixed into the ground state.

We have suggested other measurements; for example, the magnetic moment of the first and second excited $2^{+}$states. Our predictions of the sensitivity of magnetic moments to asymmetric collective motion has inspired a number experiments which we have used to extract the F-spin admixtures for the ground and $\gamma$ band in these nuclei.

i. Scissors Mode in Nuclei and Nuclear Deformation. The scissors mode in nuclei is a collective isovector orbital magnetic dipole angular oscillation of neutrons and protons against each other. This mode has been observed in electron and photon inelastic scattering from nuclei. We have derived a sum rule that relates the total magnetic dipole transition strength to this state to the nuclear deformation. We have used the experimental deformation of heavy nuclei to show that the measured orbital magnetic dipole transition strength agrees within experimental error, and have predicted this strength for nuclei not yet measured.

\section{j. Collective Motion for Proton-Rich, Neutron-Deficient, $\mathbf{N}$} $\mathbf{Z}$ Nuclei. The interacting boson model has been a successful model of the lowlying collective states of heavy nuclei. The version of the model which distinguishes between neutrons and protons (IBM-2) assumes that the low-lying collective states are composed primarily of correlated monopole, $\left(J^{\pi}=0^{+}\right)$, and quadrupole, $\left(J^{\pi}=2^{+}\right)$, pairs of protons and pairs of neutrons. IBM-2 is valid for nuclear states which have the valence protons (or neutrons) filling a major shell that is closed with respect to protons (neutrons). Such states automatically have a definite isospin. However, when the valence neutrons and protons fill the same major shell, that is $N \sim Z$, such as in light nuclei, then isospin must be introduced. To take into account isospin within the framework of boson models, a monopole and quadrupole neutron-proton pair must be included in addition to the protonproton and neutron- neutron pair in order to complete the isospin triplet. Although this IBM-3 was introduced for light nuclei, there are some medium and heavy weight proton-rich, neutron-deficient nuclei with $N \sim Z$ that have been studied and, 
with the advent of radioactive beams, there will be more such nuclei studied in the future. We have studied these nuclei by two approaches.

Since these heavy nuclei have collective low-lying states, in the first approach we determine the intrinsic motion of the collective motion in a similar manner that we did for IBM-2. Because isobaric analog states are at high excitation energy, we want an intrinsic state which has good isospin and deformation parameters that depend on isospin. We determined such a state that is a function of the quadrupole shape parameters and used it to calculate the collective energy surface of the most general isospin invariant IBM-3 Hamiltonian. We have used this energy surface to determine the possible collective motions as a function of mass number and isospin under the restriction of isospin conservation, the first time that isospin conservation has been considered in connection with collective motion of the low-lying states. We have derived formulas for the intrinsic states, excitation energies, and transition rates for the various collective bands for both axial deformed and $\gamma$ unstable nuclei as a function of isospin for the most general IBM-3 Hamiltonian that conserves isospin. The excitation modes include both symmetric and antisymmetric beta and gamma bands and also a "scissors" type isovector mode; that is, a magnetic excitation (with angular momentum $1^{+}$) in which the the protons and neutrons oscillate with respect to each other.

In the second approach, which is more quantitative, we diagonalize the isospin conserving IBM-3 Hamiltonian and determine the nuclear spectrum, eigenstates, and transition rates. As a first application, we have calculated the spectroscopy of the $N \sim Z \sim 40$ nuclei, such as the $\mathrm{Sr}$ and $\mathrm{Zr}$ isotopes. Recent measurements on these nuclei suggest that they are very deformed, but the collective motion may not be that of an axial rotor, but of a "gamma" soft rotor, a type of collective motion that is very rare in the well-studied nuclear isotopes near the valley of stability.

k. The $\Xi+\mathbf{d}->\Lambda+\Lambda+\mathbf{n}$ Reaction. There has been considerable speculation about the existence of the $H$ dibaryon, as a deeply bound six-quark state. This, in turn, has generated considerable interest in doubly strange $\Lambda \Lambda$ hypernuclei. The observation of such systems would argue against the existence of a deeply bound $H$. Therefore, $\Lambda \Lambda$ scattering information has a direct bearing on the question. Data on the neutron spectrum from $\Xi^{-} d \rightarrow \Lambda \Lambda n$ reaction should provide the best current information on $\Lambda \Lambda$ scattering. The neutron spectrum should be sensitive to the scattering length and effective range much as the photon 
spectrum from the $\pi^{-} d->\gamma n n$ reaction has been analyzed to extract the $n n$ scattering length and effective range.

We proposed to model the three-body final-state of the $\Xi^{-} d->\Lambda \Lambda n$ reaction to investigate the possibility of extracting the low-energy scattering parameters for the $\Lambda \Lambda$ interaction. We derived the separable-potential three-body equations for the $X N N$ system including the coupled-channel $\Xi^{-} d->\Lambda \Lambda n$ baryonbaryon interaction in the isospin- 0 channel. We completed $\Xi d$ elastic scattering calculations, with which we explored the structure of the elastic and total cross section as a function of the strength of the $\Lambda \Lambda$ interaction starting from an approximation to the Nijmegen one-boson-exchange potential model $D$. As a constraint upon our variation of the short-range cut-off parameter in the interaction model, we have calculated the binding energy of the ${ }_{\Lambda \Lambda}^{6} \mathrm{He}$ hypernucleus. As expected, the structure in the total cross section comes primarily from the opening of the $(\Xi N) N$ rearrangement channels. Thus, the total cross section provides little signature for the presence of a bound state in the $\Lambda \Lambda-\Xi N$ coupled channel; that is the $\Lambda \Lambda-\Xi N$ channel contribution to the cross section is small, and one must investigate carefully the neutron spectrum from the break-up reaction $\Xi^{-} d->\Lambda \Lambda n$ to determine how to unfold information about the $\Lambda \Lambda-\Xi N$ coupled channel interaction.

We have completed coding for the break-up reaction calculation and expect to obtain the first numerical results by the end of FY95. A complete numerical study utilizing the model potentials already employed in the total cross section study is expected to be completed by the end of CY95.

1. Electromagnetic Dissociation in Heavy-Ion Collisions. The availability of relativistic heavy-ion beams has opened a new avenue for the study of electromagnetic excitations of nuclei. Experiments range from single and double nucleon-removal reactions, to the study of "halo" nuclei using radioactive beams, to the possibility of multi-photon excitations of collective nuclear states. Aside from their intrinsic interest, electromagnetic excitation processes in peripheral collisions are important for determining the beam lifetime at RHIC. This facility will be one of the mainstays of experimental nuclear physics in the U.S. in the next century, and of interest to LANL experimentalists.

We initially calculated the fully quantum mechanical cross section for electromagnetic excitation of target nuclei in first Born approximation, neglecting recoil. Our final result can be expressed in terms of correlation functions and 
densities convoluted in configuration space. The result agrees with the venerable and semiclassical Weizsacker-Williams (WW) approximation for unretarded electric-dipole radiation, but our method allows an analytic evaluation of the problematic WW cutoff (contained in that result), which is determined by the details of the electric-dipole transition density. Using the Goldhaber-Teller model of that density, and uniform charge densities for both projectile and target, the cutoff was determined for the total cross section for exciting the nonrelativistic target, and found to be smaller than those used in conventional WW electric-dipole calculations. In addition, cross sections were calculated using a phenomenological momentum-space cutoff designed to model final-state interactions. For moderate projectile energies, the calculated electromagnetic dissociation cross section was found to be smaller than the semiclassical result, in qualitative agreement with experiment.

We then extended our fully quantum mechanical calculations of the electromagnetic excitation of target nuclei by relativistic heavy ions. The results for the double (simultaneous) excitation of both projectile and target nuclei in first Born approximation demonstrate that this process is negligible compared to single excitation. The tiny contribution arises largely because of small phase-space factors. The small result is comforting, since the calculations are very difficult.

We are currently completing the extension of our calculations beyond firstBorn approximation to incorporate the effects of (pure) Coulomb scattering in both initial and final states. Although Coulomb parameters on the order of 50 are possible, it is believed that Coulomb distortion effects on the excitation cross sections are very small. We have demonstrated that the Coulomb effect vanishes in leading order (high energy) and will complete our work by calculating the much more difficult sub-leading order (and presumably nonvanishing) contribution.

m. Inclusive Scattering and Euclidean Response. We have recently completed calculations of the Euclidean Response of ${ }^{3} \mathrm{H}$ and ${ }^{3} \mathrm{He}$, including the polarization observables $R_{L}^{\prime}$ and $R_{L T}^{\prime}$. The Longitudinal responses are obtained in excellent agreement with experimental results at momentum transfers of 300 and $400 \mathrm{MeV} / \mathrm{c}$. The calculations of the transverse response, however, are approximately $10 \%$ higher than experiment. The transverse response has significant corrections due to exchange currents, approximately $20 \%$ at these momentum transfers. We are continuing to explore a variety of theoretical 
uncertainties in these calculations, including model dependence in the exchange currents and more accurate relativistic calculations.

n. Path Integral Calculations of $\mathbf{A}=6$ and 7. We have completed the first accurate microscopic calculations of the low-lying states in the 6-body system. These calculations indicate that a realistic nuclear interaction provides an accurate description of the $A=6$ ground states and low-lying spin excitations. We have now also incorporated an exact two-body propagator into these calculations, resulting in a speed increase of roughly a factor of 5 . This new method will allow us to perform more accurate calculations of ground-state expectation values and low-energy transitions, and should enable us within the next two years to proceed with calculations of 7- and 8-body systems. We are also exploring "constrainedpath" alternatives for approximate calculations of nuclear systems.

\section{Invited Talks}

C. J. Benesh, "Quark Model Calculations of Symmetry Breaking in Parton Distributions", presented at the International Symposium and Workshop on Particle Theory and Phenomenology, Iowa State University, May 17-26, 1995.

J. Carlson, "Recent Progress in Path Integral Calculations of Nuclei", 15th European Few-Body Conference, Peniscola, Spain, June 1995.

J. L. Friar, "What Can We Learn from Multi-Nucleon Breakup Processes", Invited talk presented at Spring Meeting of the American Physical Society, Washington, DC, April 22, 1994.

J. L. Friar, "Few-Body Physics -- Then and Now", Summary talk presented at 24th International Conference on Few-Body Problems in Physics, Williamsburg, VA, May 31, 1994.

J. L. Friar, "Chiral Symmetry in Nuclei", Invited talk presented at International Workshop on Chiral Dynamics in Hadrons and Nuclei, Seoul, South Korea, February 6-11, 1995. 
J. L. Friar, "Nuclear Forces and Chiral Theories", Invited talk presented at 15th European Conference on Few-Body Problems in Physics," Peniscola, Spain, June 5-9, 1995.

B. F. Gibson, "Strangeness Physics", Workshop on Future Directions in Particle and Nuclear Physics at Multi-GeV Hadron Beam Facilities," (FNAL, December 1994), LA-UR-94-4215.

J. N. Ginocchio, "Eikonal Scattering from Complex Systems," invited talk at the American Mathematical Society meeting, Springfield, Missouri, March 20-21, 1992.

J. N. Ginocchio, "Testing Generalized Seniority and the Interacting Boson Model with Double Charge Exchange", Workshop on Algebraic Techniques for Quantal Systems, Jan. 23, 1993, University of Pennsylvania, Philadelphis, PA.

J .N. Ginocchio, "Nuclear Collective Motion with Isospin", invited talk at the International Conference on Perspectives in the Interacting Boson Model, Padova, Italy, June 11-17, 1994.

J .N. Ginocchio, "New Types of Collective Motion for Heavy Nuclei with $\mathrm{N} \sim \mathrm{Z}$ ", Intel Workshop on Spin and Isospin Excitation in Nuclear Systems, European Center for Theoretical Physics, Trento, Italy, April 3, 1995.

P. Herczeg, "Left-Right Symmetric Models and Their Phenomenological Implications", at the 4th International Symposium on Weak and Electromagnetic Interactions in Nuclei (WEIN '95), Osaka, Japan, June 12-16, 1995.

P. Herczeg, "Muon Decay Beyond the Standard Model", at the Annual General Meeting of the TRIUMF Users Group, TRIUMF, Vancouver, Canada, December 6, 1994.

P. Herczeg, "Searches for T-Odd Interactions in Nuclear Processes: Review of the Theory", the Moriond '95 Workshop on Dark Matter in Cosmology, Clocks and Tests of Fundamental Laws, Villars-sur-Ollon, Switzerland, January 21-28, 1995. 
P. Herczeg, "Extensions of the Minimal Standard Model", at the Town Meeting on Electroweak Interactions, Astrophysics and Non-Accelerator Experiments, LBL, Berkeley, California, February 4-5, 1995.

P. Herczeg, "Beta Decays and New Interactions", invited talk at the workshop on Future Directions in Particle and Nuclear Physics at Multi-GeV Hadron Beam Facilities, Brookhaven, March 4, 1993.

P. Herczeg, "Beta Decay Beyond the Standard Model", invited talk at the workshop on Symmetry Tests in Semileptonic and Leptonic Weak Interactions, Louvain-la-Neuve, Belgium, June 2-4, 1993.

\section{Publications}

1. Ahluwalia, D.V., T. Goldman, and M.B. Johnson, " $(\mathrm{j}, 0)+(0, \mathrm{j})$ Representation Space: Majorana-Like Construct," Acta Phys. Pol. B 25, 1267 (1994).

2. Burdman, G., T. Goldman, and D. Wyler, "Radiative Leptonic Decays of Heavy Mesons," Phys. Rev. D 51, 111 (1995).

3. Carlson, J., "Recent Progress in Path Integral Calculations of Nuclei" (to be published in Few Body Systems).

4. Forest, J.L., V.R. Pandharipande, J. Carlson, et al., "Variational Monte Carlo Calculations of ${ }^{3} \mathrm{H}$ and ${ }^{4} \mathrm{He}$ with a Relativistic Hamiltonian-II," Phys. Rev. C 52, 576 (1995).

5. Forest, J.L., V.R. Pandharipande, and J.L. Friar, "Relativistic Nuclear Hamiltonians," Phys. Rev. C 52, 568 (1995).

6. Friar, J.L., "Dimensional Regularization and Nuclear Potentials" (submitted to Phys. Rev. C).

7. Friar, J.L. and S.A. Coon, "The Leading-Order Nuclear Pi-GammaExchange Force" (submitted to Phys. Rev. C).

8. Friar, J.L. and G.L. Payne, "Assessment of Triton Potential Energy" (to be published in Few Body Systems).

9. Friar, J.L. G.L. Payne, W. Glockle, et al., "Benchmark Solutions for N-D Breakup Amplitudes," Phys. Rev. C 51, 2356 (1995). 
10. Ginocchio, J.N., "The Fractional Quantum Hall Effect and the Spherical Shell Model, from Spectroscpy to Chaos," in From Spectroscopy to Chaos,

A. Das and D.S. Koltun, Eds. (World Scientific, Singapore, 1994).

11. Ginocchio, J.N. and C.W. Johnson, "Hermitian Boson Mapping and Finite Truncation," Phys. Rev. C 50, R571 (1994).

12. Ginocchio, J.N. and A. Leviatan, "Quadrupole Collectivity with Isospin," Phys. Rev. Lett. 73, 1903 (1994).

13. Ginocchio, J.N., P. von Neumann-Cosel, and A. Richter, "Relation between the Scissors Mode and the Interacting Boson Model Deformation" (submitted to Phys. Rev. Lett.).

14. Goldhaber, A.S. and T. Goldman, "Confinement, Crossing Symmetry, and Glueballs," Phys. Rev./ Lett. B 344, 319 (1995).

15. Pudliner, B.S., V.R. Pandharipande, J. Carlson, et al., "Quantum Monte Carlo Calculations for a $\leq 6$ Nuclei," Phys. Rev. Lett. 74, 4396 (1995).

16. Ping. J., F. Wang, and T. Goldman, "An Extension of Fractional Parentage Expansion to the Nonrelativistic and Relativistic Three Flavor Unitary Group Dibaryon Calculations," Phys. Rev. C 51, 1648 (1995).

17. Redman, J. and R.R. Silbar, "Experimental Physics, Selecting an Operating System, Part II: NEXSTEP," Comput. Phys. 9, 261 (1995).

18. van Kolck, U., J.L. Friar, and T. Goldman, "Phenomenological Aspects of Isospin Violation in the Nuclear Force" (submitted to Phys. Rev. Lett.).

19. Wang, F., G.H. Wu, L.J. Teng, et al., "Quark Delocalization, Color Screening, and Nuclear Intermediate Range Attraction $\Pi$," Mod. Phys. Lett. A 10, 1895 (1995).

20. Wang, F., G.H. Wu, L.J. Teng, et al., "Quark Delocalization, Color Screening, and Dibaryons," Phys. Rev. C 51, 3411 (1995).

21. Zhang, S., J. Carlson, and E. Gubernatis, "A Constrained Path Quantum Monte Carlo Method for Fermion Ground States," Phys. Rev. Lett. 74, 3652 (1995).

\section{DISCLAIMER}

This report was prepared as an account of work sponsored by an agency of the United States Government. Neither the United States Government nor any agency thereof, nor any of their employees, makes any warranty, express or implied, or assumes any legal liability or responsibility for the accuracy, completeness, or usefulness of any information, apparatus, product, or process disclosed, or represents that its use would not infringe privately owned rights. Reference herein to any specific commercial product, process, or service by trade name, trademark, manufacturer, or otherwise does not necessarily constitute or imply its endorsement, recommendation, or favoring by the United States Government or any agency thereof. The views and opinions of authors expressed herein do not necessarily state or reflect those of the United States Government or any agency thereof. 